\title{
Emerging Applications of Optical Coherence Tomography Angiography (OCTA) in neurological research
}

\author{
Liang Wang ${ }^{1}$, Olwen Murphy ${ }^{2}$, Natalia Gonzalez Caldito ${ }^{2}$, Peter A. Calabresi ${ }^{2}$ and Shiv Saidha ${ }^{2 *}$ (D)
}

\begin{abstract}
Purpose: To review the clinical and research value of optical coherence tomography angiography (OCTA) in the field of neurology.

Methods: Current literature involving OCTA were reviewed through PubMed using the search terms "optical coherence tomography angiography", with "multiple sclerosis", "Alzheimer's disease", "optic neuropathy", or other closely-related terms.

Results: OCTA has been applied in research to advance our understanding of the pathobiology of neurological disorders. OCTA-derived blood flow and vessel density measures are altered in multiple sclerosis (MS), Alzheimer's disease (AD), and various optic neuropathies (ON) in varying regions of the posterior segment vasculature of the eye. These emerging research findings support the occurrence of retinal vascular alterations across a host of neurological disorders and raise the possibility that vasculopathy can be clinically relevant since it contributes to the pathobiology of several neurological disorders.

Conclusion: OCTA may be beneficial for neurological research. Additional investigations using OCTA in neurological disorders will help to further validate its clinical and research utilities in terms of characterizing the role of vasculopathy in neurological disorders.
\end{abstract}

Keywords: Optical coherence tomography angiography, Neurology, Multiple sclerosis, Alzheimer's disease, Optic neuropathy

\section{Background}

Optical coherence tomography angiography (OCTA) enables visualization of the ocular microvasculature through non-invasive, high-resolution enface and depth resolved imaging [1]. First adapted from OCT and approved by the Food and Drug Administration (FDA) in 2015, OCTA provides structural and potentially functional information regarding the superficial and deep retina, as well as choroidal vasculature [2]. Vessel alterations including but not limited to neovascularization [3], capillary loss [4], non-perfusion [5], increase in vessel tortuosity [6], and decrease in relative flow rates [7] have been reported with OCTA in various

\footnotetext{
* Correspondence: ssaidha2@jhmi.edu

${ }^{2}$ Division of Neuroimmunology and Neurological Infections, Johns Hopkins

Hospital, 600 N. Wolfe St., Baltimore, MD 21287, USA

Full list of author information is available at the end of the article
}

ocular pathological conditions such as age related macular degeneration [8], macular telangiectasia [9], artery and vein occlusions [10,11], diabetic retinopathy [12], and glaucoma [7]. Undetectable with commonly utilized ophthalmic tools such as traditional OCT and fundus photography, deeper, finer and more subtle vascular alterations are traditionally visualized through intravenous fluorescein angiography (IVFA), which requires the injection of a fluorescence dye [11]. However, leakage of the dye related to the breakdown in the blood-retinal-barrier has been reported to potentially obscure capillary loss, vessel distortion, and early neovascularization $[13,14]$. While indocyanine green angiography (ICGA) can potentially better visualize deeper regions of the chorioretinal vasculature, this technique still employs contrast-based injections [15-17]. Since OCTA may allow these obscured structures to be more clearly distinguished, and non-invasively without the use of contrast agents, the

(C) The Author(s). 2018 Open Access This article is distributed under the terms of the Creative Commons Attribution 4.0 International License (http://creativecommons.org/licenses/by/4.0/), which permits unrestricted use, distribution, and reproduction in any medium, provided you give appropriate credit to the original author(s) and the source, provide a link to the Creative Commons license, and indicate if changes were made. The Creative Commons Public Domain Dedication waiver (http://creativecommons.org/publicdomain/zero/1.0/) applies to the data made available in this article, unless otherwise stated. 
development of OCTA represents a major contribution towards advancing ophthalmic imaging in ocular disease diagnosis, monitoring and treatment $[17,18]$. While OCTA utilization has been growing in ophthalmic clinical practices, a potential application of OCTA in the field of neurology has also been under active investigation. Benefiting from OCTA's depth imaging capabilities, pial vessels in the cerebral microvasculature of mouse models can still be visualized in the setting of intact scalps [19]. OCTA has also enabled rodent models of stroke and traumatic brain injury (TBI) to be studied in vivo. The real time analysis OCTA allows of the structural and potential functional alterations within the microvasculature have helped to further characterize disease complexes and more specifically, vascular components underlying the pathobiology of diseases, which could open up new therapeutic targets and avenues of clinical monitoring [20,21]. Due to the lack of direct access, human cerebral microvascular alterations are difficult to non-invasively monitor in vivo. However, the brain can possibly be interrogated through the eyes since the vasculature of the retina and brain is similar in its anatomy and physiology [22, 23]. Furthermore, through the process of trans-synaptic degeneration, cerebral tissue injury may result in tissue degeneration of the optic nerves and retinal ganglion cells, which are supplied by the central retinal artery [24]. Thus, structural and functional changes in the optic nerve and retina may hypothetically result in ocular vascular alterations. Using OCTA, non-invasive in vivo studies of neurological disorders through the human eye have emerged for multiple sclerosis (MS) [25-28], Alzheimer's disease (AD) [29-31], and various optic neuropathies $(\mathrm{ON})$ [32-36].

\section{Algorithms and analysis}

Adapted from traditional OCT, a powerful non-invasive imaging tool for visualizing and segmenting the discrete layers of the retina, OCTA enables high-speed imaging acquisition of the retinal vasculature for angiography. Analysis of the retinal vasculature can be completed through a number of algorithms. Ultrahigh-sensitive optical microangiography (OMAG, Cirrus HD-OCT 5000 ${ }^{\mathrm{m}}$, Carl Zeiss Meditec. Inc) [37], split-spectrum amplitude decorrelation angiography (SSADA, Optovue RTVue XR Avanti ${ }^{\text {im }}$, Optovue Inc., Fremont, CA) [38], OCT Angiography Ratio Analysis (OCTARA, Topcon DRI OCT Triton Swept source OCT $^{\mathrm{Tm}}$, Topcon, Japan), and full spectrum amplitude decorrelation algorithm (FS-ADA, Spectralis OCT2 module prototype ${ }^{\mathrm{rm}}$, Heidelberg Engineering, Germany) [39] have been commonly used in OCTA studies. These algorithms produce three dimensional, $3 \times 3 \mathrm{~mm}^{2}$ A lines in cross-sectional images or 1 pixel in the enface image in less than $3 \mathrm{~s}$. The A scan (line in the cross-sectional image, 1 pixel in the enface image) has a scan rate of $68 \mathrm{kHz}$ with OMAG, $70 \mathrm{kHz}$ with SSADA, $100 \mathrm{kHz}$ with
OCTARA, and $85 \mathrm{kHz}$ with FS-ADA. The A scans make up B scans. The B scan is taken four times in the same region with OMAG and OCTARA. Two consecutive $B$ scans are taken with SSADA and only one B scan is required with FS-ADA [40]. Images with a field of view larger than $6 \times 6 \mathrm{~mm}^{2}$ can also be acquired using commercially available systems, but they often have a lower lateral resolution with individual A-scans covering the scan areas. Images with larger fields of view and relatively higher lateral resolution can be acquired using the high speed Swept Source (SS) OCTA system [41, 42] or through wide-field montages (Fig. 1) [37].

To detect red blood cell movement in OCT images, repeated B-scans are taken at the same cross-section of the same location with similar scanning methods used in other OCTA systems (Fig. 2) [2, 42, 43]. Changes in the phase and intensity of the OCT signal can then be detected through the motion of red blood cells. OMAG utilizes both of the potential changes to generate angiograms with comparably high image contrast and vessel connectivity. Alternatively, SSADA utilizes the signal intensity through decorrelation values between consecutive B-scans where increased red blood cell movement resulted in increased decorrelation values [2]. Thus, areas of low decorrelation may indicate regions of nonperfusion and/or vessel loss. This algorithm decreases the effects of phase noise that may occur especially when the light source is unstable. The signal is also further processed to improve vessel detection and remove noise introduced by motion [38]. Instead of decorrelation values, OCTARA uses intensity ratio analyses, which helps increase the axial resolution by not splitting the spectrum. On the other hand, FS-ADA can be used to analyze blood flow through motion contrast [40].

Owing to the fact that OCTA has traditional OCT function that can be used to acquire information on retinal structure, multiple layers can be identified so that the vasculature in the corresponding layers can be segmented [44]. For example, in Optovue ${ }^{\mathrm{Tw}}$, the OCT scan can be segmented into a number of composite layer regions, including the inner retina (from ganglion cell layer to inner plexiform layer), middle retina (from inner nuclear layer to outer plexiform layer), outer retina (from outer nuclear layer to external limiting membrane), choriocapillaris, and choroid [8]. These regions on the OCTA can be analyzed for a number of vascular features. For instance, the presence of neovascularization [45], an increase in tortuosity [6], and areas of capillary loss [46] can be qualitatively analyzed. Additional features such as the area of the foveal avascular zone (FAZ), parafoveal region, regions of non-perfusion and relative density can be quantitatively determined either through the fractal analysis or pixel counting methods. The flow rate is the average decorrelation value of 


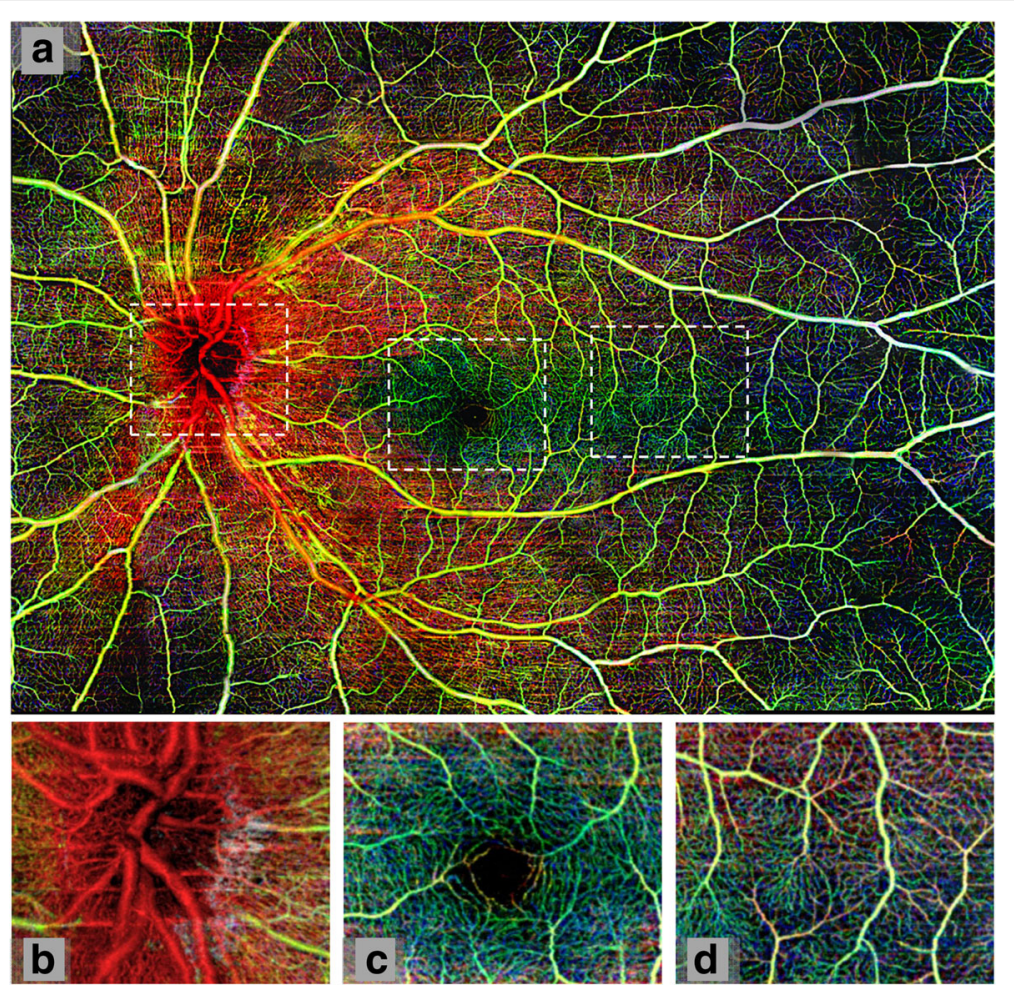

Fig. 1 Wide field montage of healthy retinal microvasculature. Using Cirrus ${ }^{\mathrm{TM}}$ optical coherence tomography angiograph with optical microangiography, 42 scans were taken and combined to create a $12 \times 16 \mathrm{~mm}^{2}$ image (panel a). The images are portrayed in different colors representing varying retinal depth. Red depicts the retinal nerve fiber layer, green the ganglion cell and inner plexiform layers, and blue the inner nuclear and outer plexiform layers. White boxes outline (b) optic nerve head, (c) fovea, and (d) temporal region. (Reprinted from Zhang et al. Wide-field imaging of retinal vasculature using optical coherence tomography-based microangiography provided by motion tracking. JBO, 20, 066009 (2015), DOl: https://doi.org/10.1117/1.JBO.20.6.066008) [43]

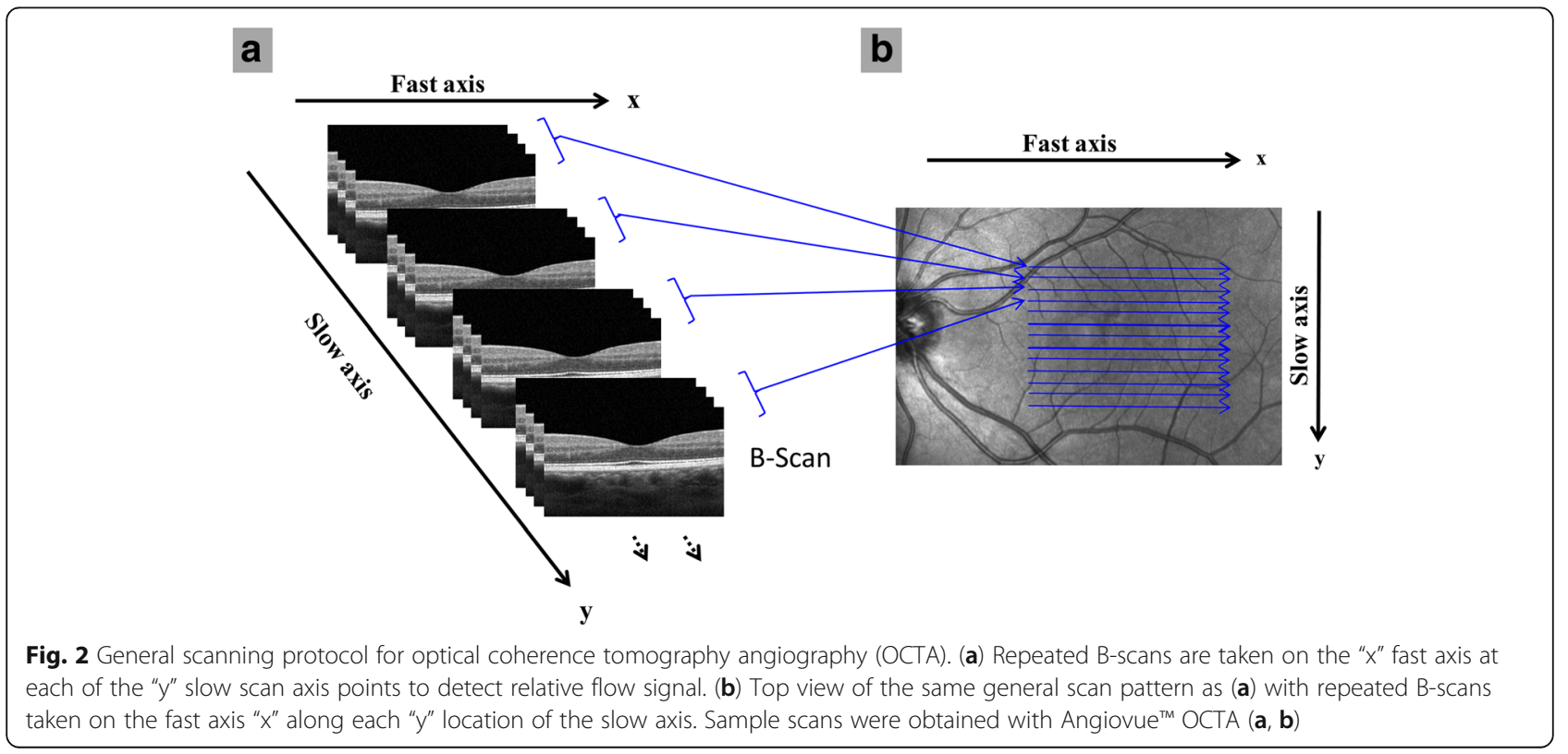


sequential B-scans on OCTA, which can be used as a surrogate for blood flow rates [47]. Furthermore, Optovue ${ }^{\text {tw }}$ includes an analysis package in its system that automatically analyzes the FAZ and vessel density in different retinal sub-regions.

\section{Healthy eyes}

The eye enables one of the dominant methods of perception by focusing light stimuli through the anterior segment onto the posterior segment, which includes the photosensitive cells in the retina that are responsible for converting light stimuli into electrochemical impulses, which are subsequently sent to the brain through the optic nerve [24]. Using OCTA, the vascular plexuses of the inner retina, outer retina, and choriocapillaris can be non-invasively visualized. Supplying the retina, one of the regions of the body with the highest metabolic demand, the inner retinal vascular plexus is further separated into the superficial vascular plexus (SVP), which supplies the retinal nerve fiber layer, andganglion cell layer, and the deep vascular plexus (DVP), which supplies the inner plexiform layer, inner nuclear layer, and outer plexiform layer (Fig. 3) [48]. In healthy eyes, OCTA cross sectional images show clear retinal and choroidal layers with relatively even blood flow, corresponding with en face angiograms that show healthy retinal and choroidal layers with dense and well connected vasculatures [38]. The en face angiograms also show normal avascular regions in the vitreous above the inner limiting membrane and in the outer retina between the outer plexiform layer and Bruch's membrane. OCTA findings of microvascular structure, including capillary patterns of the perifoveal and peripapillary regions have been verified through comparison to examined post-mortem tissue in histology studies [14].

OCTA findings in healthy volunteers have been critical for forming a solid foundation for the normative retinal and choroidal microvasculature, determining when abnormalities are present, and will ultimately play a central role in helping to guide future disease characterization and diagnosis. The mean relative parafoveal flow (flow index) and relative vessel coverage (decorrelation values) have been reported to significantly decrease with normal aging, while the FAZ area (determined by pixel counting) significantly increases in size with normal aging [49]. The correlation between vessel and perfusion loss with increasing age in healthy vasculatures have been reported in prior studies using blue field stimulation [50], magnetic resonance imaging [51], and IVFA [52]. However, analyses of the FAZ determined by fractal analysis, as opposed to pixel counting, did not find significant changes in the FAZ with age, which might be partially due to unadjusted

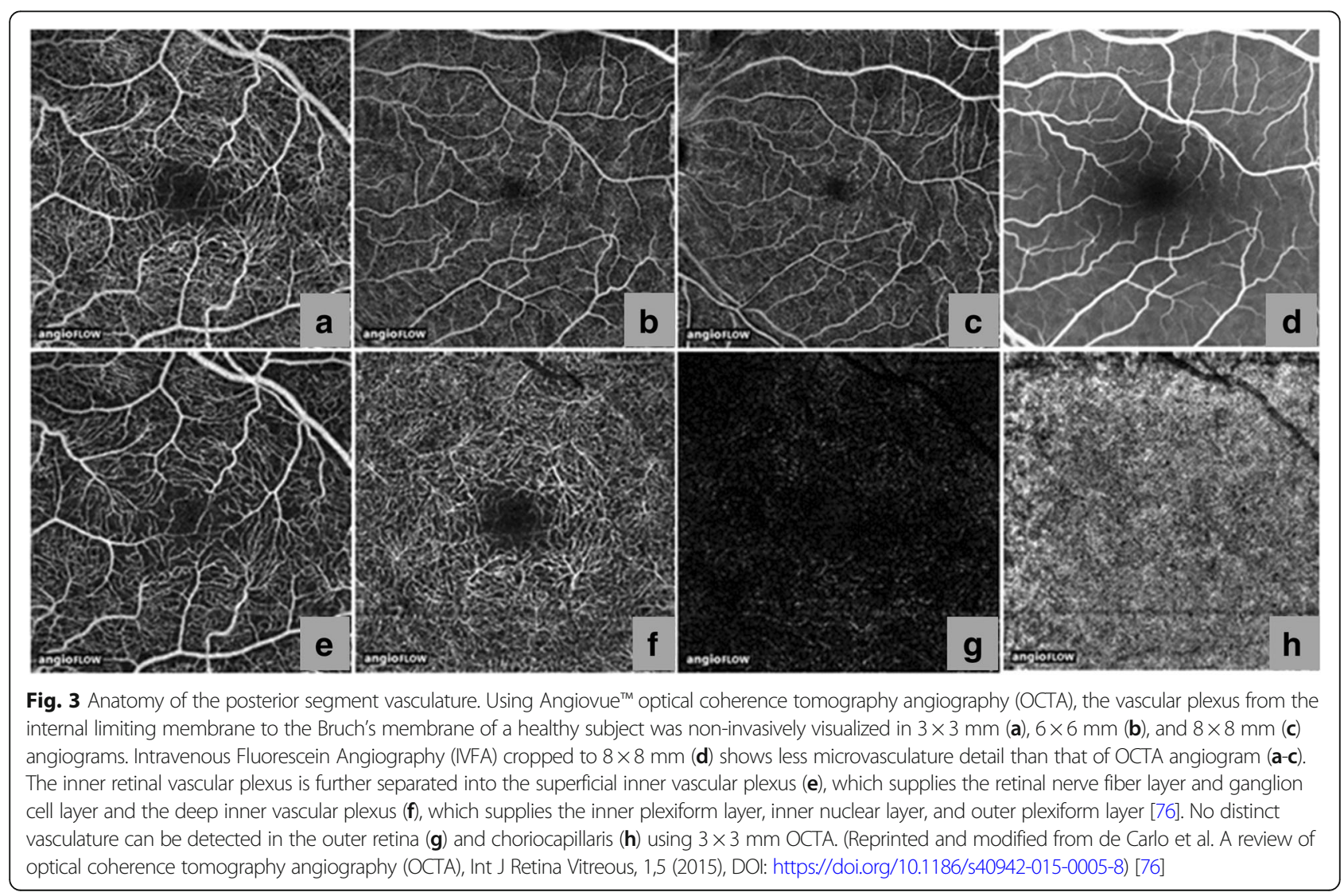


magnification error [53]. However, fractal dimension showed a relatively denser DVP in comparison to the SVP in relation to age-related changes $[18,54]$. These findings are supported by previous research that analyzed human donor eyes through confocal microscopy in the perifoveal region [55]. The FAZ area, which varies in size from person to person has been reported to be on average $0.32 \pm 0.11 \mathrm{~mm}^{2}$ in size through pixel counting on angiograms generated with SSADA in a study of healthy volunteers $(n=144$, age range 10-79 years) [56].

\section{Multiple sclerosis}

Multiple Sclerosis (MS) is a chronic inflammatory demyelinating disorder of the central nervous system (CNS) where neurodegeneration primarily occurring because of inflammation is thought to be the principal substrate underlying disability. Post-mortem studies demonstrate that up to $99 \%$ of MS patients exhibit demyelinating plaques within their optic nerves, making optic nerve involvement a ubiquitous part of the MS disease process $[57,58]$. Optic neuritis $(\mathrm{ON})$ is the initial manifestation of MS in approximately $25 \%$ of patients and occurs in approximately $50 \%$ of patients at some point in the disease course [28]. Since available treatments generally target the autoimmune and inflammatory aspects of MS and vary in effectiveness across patients [27], sensitive biomarkers for monitoring disease progression and therapeutic efficacy are desperately needed. OCT-derived retinal measures have emerged for this purpose, with the retina representing the most accessible component of the CNS. OCT identified thinning of the retinal nerve fiber layer (RNFL) and composite of the ganglion cell and inner plexiform layers (GCIP) in particular have become biomarkers of global CNS neurodegeneration in MS [59].

OCTA now shows utility in detecting and studying damage to the retinal vasculature in MS. The parafoveal and optic nerve head (illustrated in Fig. 4) flow index, a representation of relative blood flow velocity in the vasculature, was determined to be significantly lower in MS eyes with a history of ON (MSON) in comparison to eyes without a history of ON (MSNON), as well as relative to eyes in healthy controls [25, 28] (Table 1). Furthermore, significant vessel reduction in the superficial and deep retinal vascular plexuses in the parafoveal area was found in both MSON and MSNON eyes as compared to healthy control eyes [26, 27]. Retinal vascular density was correlated with retinal layer thicknesses (RNFL, GCIP, total macular volume and inner nuclear layer) and increased disability in MS patients as determined through Expanded Disability Status Scale scores [27].

The etiology underlying the decrease in blood flow and density of the retinal vasculature in MS patients remain unclear. Accumulating evidence has determined that inflammation leads to loss of neurons in the inner retina. The reduced number of neurons, resulting in reduced metabolic demand, may result in reduced blood supply provided by the vessels of the SVP, which in turn supplies the DVP through anastomoses [25, 26]. Inflammation is thought to lead to mild alterations in vascular function, including endotheliopathy, and postulated to result in decreased perfusion and neuronal damage [60], although this pathologic disease mechanism remains to be definitively demonstrated. Interestingly, studies of the cerebral microvasculature in MS have revealed possible hypoperfusion in the normal appearing gray matter and white matter $[61,62]$. A recent cross-sectional study of the Retinal Function Imager, an ophthalmic multimodal imaging modality, in relapsing remitting MS (RRMS) $(n=17)$ revealed reductions in retinal arteriolar and venular blood flow velocities as compared to healthy controls, although determinations with respect to underlying mechanisms of the observed findings or their relationships to ON history were not possible [63]. In MS, it is

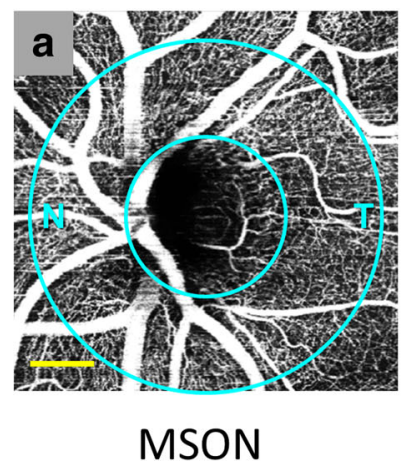

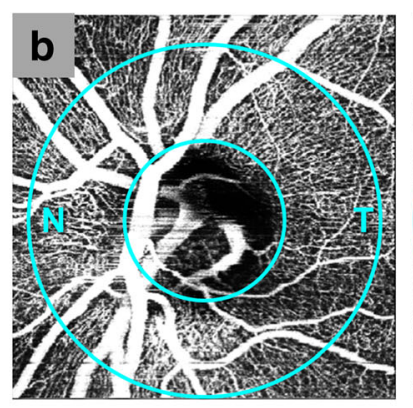

MSNON

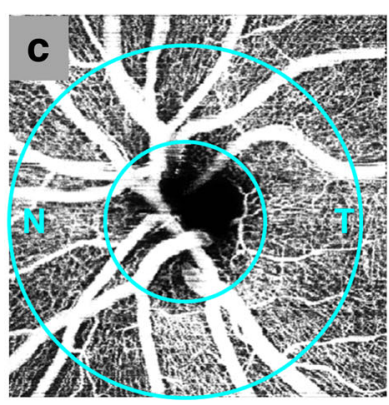

Control

Fig. 4 Optical coherence tomography angiography (OCTA) of the optic nerve head (ONH) in a representative Multiple Sclerosis (MS) patient. As determined with split-spectrum amplitude decorrelation angiography (SSADA), images ( $N=$ nasal, $T=$ temporal) show apparent qualitative reduction of the ONH microvascular density in the peripapillary area (between circles) predominantly in the temporal region in both MS eyes with a history of ON (MSON) (a) and MS eyes without a history of ON (MSNON) (b) eyes in comparison to a healthy control example (c). Bar $=0.5 \mathrm{~mm}$ 
Table 1 Summary of significant optical coherence tomography angiography findings in neurological disorders *

\begin{tabular}{|c|c|c|c|c|c|c|c|c|}
\hline Author & Parameter & Disorder & $N$ (eyes) & Measurement & Compare & $N$ (eyes) & Measurement & $\%$ Difference \\
\hline Wang et al. (2014) [25] & flow index - $\mathrm{ONH}$ & MSON & 14 & $0.140 \pm 0.020$ & $\mathrm{HC}$ & 21 & $0.160 \pm 0.010$ & $-14.3 \%$ \\
\hline Spain et al. (2017) [28] & flow index - ONH & MSON & 25 & N/A & $\mathrm{HC}$ & 55 & $\mathrm{~N} / \mathrm{A}$ & $-14.7 \%$ \\
\hline \multirow[t]{4}{*}{ Feucht et al. (2017) [26] } & vessel density \% - SVP & MSON & 16 & 50 & $\mathrm{HC}$ & 100 & 54 & $-4.0 \%$ \\
\hline & vessel density $\%$ - SVP & MSON & 16 & 50 & MSNON & 56 & 53 & $-3.0 \%$ \\
\hline & vessel density \% - DVP & MSON & 16 & 62 & $\mathrm{HC}$ & 100 & 64 & $-2.0 \%$ \\
\hline & vessel density \% - DVP & MSON & 16 & 62 & MSNON & 56 & 64 & $-2.0 \%$ \\
\hline \multirow[t]{2}{*}{ Lanzillo et al. (2017) [27] } & vessel density $\%$ - parafovea is & MSON & 23 & $50.96 \pm 5.33$ & MSNON & 77 & $51.71 \pm 5.82$ & $-1.5 \%$ \\
\hline & vessel density $\%$ - macula & MS & 100 & $48.71 \pm 4.44$ & $\mathrm{HC}$ & 92 & $53.08 \pm 3.31$ & $-9.0 \%$ \\
\hline \multirow[t]{2}{*}{ Bulut et al. (2017) [29] } & $\operatorname{area}\left(m m^{2}\right)-F A Z$ & $A D$ & 26 & $0.47 \pm 0.18$ & $\mathrm{HC}$ & 26 & $0.33 \pm 0.08$ & $29.8 \%$ \\
\hline & vessel density $\%$ - macula & $\mathrm{AD}$ & 26 & $45.50 \pm 3.85$ & $\mathrm{HC}$ & 26 & $48.67 \pm 3.29$ & $-7.0 \%$ \\
\hline \multirow[t]{3}{*}{ Jiang et al. (2017) [31] } & vessel density - SVP & $A D$ & 12 & 1.68 & $\mathrm{HC}$ & 21 & 1.74 & $-3.6 \%$ \\
\hline & vessel density - DVP & $A D$ & 12 & 1.65 & $\mathrm{HC}$ & 21 & 1.72 & $-4.2 \%$ \\
\hline & vessel density - DVP SN & $\mathrm{MCl}$ & 19 & 1.54 & $\mathrm{HC}$ & 21 & 1.62 & $-5.2 \%$ \\
\hline Fard et al. (2018) [33] & vessel density $\%$ - $P C$ & NAION & 31 & $30.1 \pm 6$ & $\mathrm{HC}$ & 77 & $42.3 \pm 2.3$ & $-40.5 \%$ \\
\hline Song et al. (2017) [36] & vessel density $\%$ - PC & NAION & 41 & $52.07 \pm 7.68$ & $\mathrm{HC}$ & 30 & $58.68 \pm 3.16$ & $-12.7 \%$ \\
\hline Ling et al. (2017) [35] & nonperfusion area $\%$ - ONH & NAION & 21 & $17.84 \pm 6.18$ & $\mathrm{HC}$ & 19 & $8.61 \pm 1.65$ & $51.7 \%$ \\
\hline
\end{tabular}

Abbreviations: $O N H=$ optic nerve head; $S V P=$ superficial vascular plexus; $D V P=$ deep vascular plexus; $I S=$ inferior sector; $F A Z=$ foveal avascular $z o n e ; S N=$ superior nasal quadrant; $P C=$ peripapillary capillaries; $M S O N=$ Disorder: multiple sclerosis with a history of optic neuritis; $M S=$ multiple sclerosis with and without history of optic neuritis; $A D=$ Alzheimer's disease; $M C l=$ mild cognitive impairment; $N A I O N=$ non-arteritic anterior ischemic optic neuropathy; $H C=C o m p a r e:$ healthy controls; $M S N O N=$ multiple sclerosis without a history of optic neuritis

* Listed comparisons were significantly different $(P<0.05)$

also recognized that elevations in hypoxia inducible factors (indicating vascular compromise) occur in regions of neurodegeneration [64]. Future studies employing easily performed OCTA with larger sample sizes, longitudinal follow up, and more focus on potential correlations to other MS biomarkers in the CNS will help further characterize aberrations in the retinal vasculature in MS and provide insights into MS disease mechanisms and even novel therapeutic strategies.

\section{Alzheimer's disease}

Alzheimer's disease (AD), the most common cause of dementia [65], is a progressive neurodegenerative disorder that is more common with aging and characterized by an accumulation of misfolded protein, in particular amyloid-beta $(\mathrm{AB})$ and neurofibrillary tangles (NFTs) [29]. Prior to AD onset, patients often have mild cognitive impairment (MCI), during which cognitive function declines, but patients have sufficient cognitive function to perform their activities of daily living [66]. $\mathrm{AD}$ has been associated with a number of vascular risk factors including stroke, diabetes mellitus, atherosclerosis, and hypertension [67]. Moreover, cerebral hypoperfusion, increased cerebral vascular tortuosity, and decreased vascular density have been reported in AD patients $[29,68]$, which may indicate the presence of a vasculopathy in the pathogenesis of AD. This probable disease mechanism, while difficult to analyze directly due to the location of the brain within the skull, may be investigated through the retinal microvasculature. The retinal and cerebral vasculatures share similar embryonic, physiologic, and anatomic features $[30,63]$. Furthermore, similar to the brain, $A B$ accumulation was found in the retina in $A D$ [69]. Using OCT, thinning of the GCIP in the retina was suggested to be a potential biomarker of neurodegeneration and disease severity in $\mathrm{AD}$ [70].

Further assessments of the individual vessel plexuses of the retina are possible through OCTA. All regions of the SVP and DVP in AD patients were found to exhibit significantly lower retinal vascular density when compared to healthy controls (Fig. 5) [29, 31]. The loss of retinal vessel density could be an indication of the increased accumulation of $A B$ in the retina [69], which can theoretically confine vascular endothelial growth factor (VEGF) within amyloid plaques and potentially reduce angiogenesis [29]. Furthermore, significant enlargement of the foveal avascular zone (FAZ) formed by the retinal plexuses was observed using OCTA in AD, which is a potential sign of ischemia and in itself may contribute to reducing retinal vessel density [29]. However, only DVP loss has been found to be significantly correlated with GCIP thinning in AD. This may be related to the relatively larger vessels within the SVP that may be less sensitive to disease progression in comparison to those of the DVP [31]. The retinal microvasculature may be a sensitive marker for cerebral microvasculature 
alterations, which could be useful for monitoring the progression of AD. In summary, OCTA analysis of the retinal microvasculature revealed significant loss of vessel density in $\mathrm{AD}$ patient eyes in comparison to eyes from normal controls and patients with MCI [31]. Future studies with larger patient cohorts may help to establish the retinal vasculature, as imaged by OCTA, as a biomarker of $\mathrm{AD}$, predictor of conversion from $\mathrm{MCI}$ to $\mathrm{AD}$, and a tool for investigating the effectiveness of putative $\mathrm{AD}$ treatments.

\section{Optic neuropathy}

\section{Anterior ischemic optic neuropathy}

Characterized by severe vision loss due to ischemia of the small vessels supplying the anterior portion of the optic nerve head, anterior ischemic optic neuropathy (AION) can be classified as arteritic (AAION) or non-arteritic (NAION) [35]. Accounting for up to $15 \%$ of AION cases, AAION is caused by inflammation within arteries primarily as a result of underlying giant cell arteritis (temporal arteritis) [32]. NAION is the more common form of AION and accounts for approximately $85 \%$ of AION cases. NAION is considered a form of small vessel disease, which usually occurs in patients with various risk factors such as hypertension, diabetes, or dyslipidemia. These factors are unrelated to inflammation [32]. Furthermore, there is no consistently effective treatment method to prevent irreversible vision loss in NAION. While OCTA cannot differentiate between AAION and NAION, the regions of non-perfusion with ischemic boundaries can be clearly identified on OCTA of the optic disk [32].

OCTA has shown promise in monitoring NAION progression through sectorial peripapillary capillary density reduction [32, 33] (Fig. 6) and increased non-perfusion area percentages. The non-perfusion area was shown to be significantly increased in NAION eyes in comparison to healthy control eyes, and correlates with the severity of peripheral visual field loss [35] and central vision impairment (as assessed by best corrected visual acuity) [36]. Furthermore, significant reduction of the RNFL and GCIP [71] in the macular region have been reported in NAION eyes assessed by OCT. Since decreased optic disc perfusion detected via OCTA shows correlation

\section{Superficial Vascular Plexus}
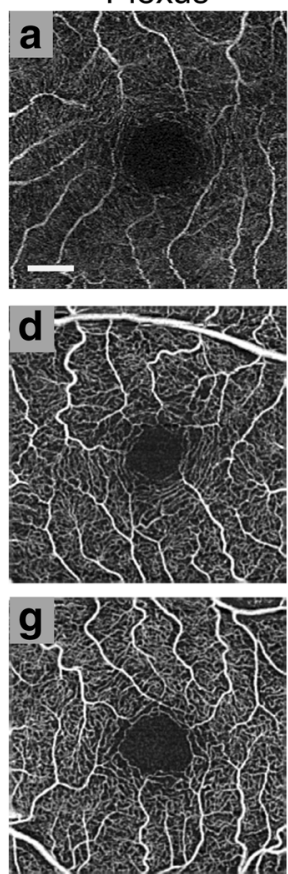
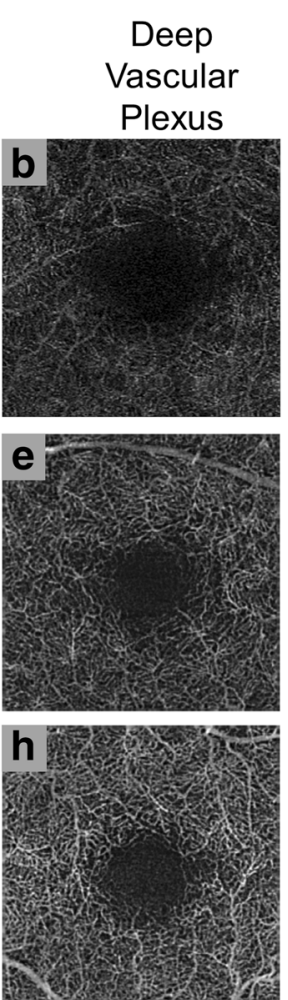

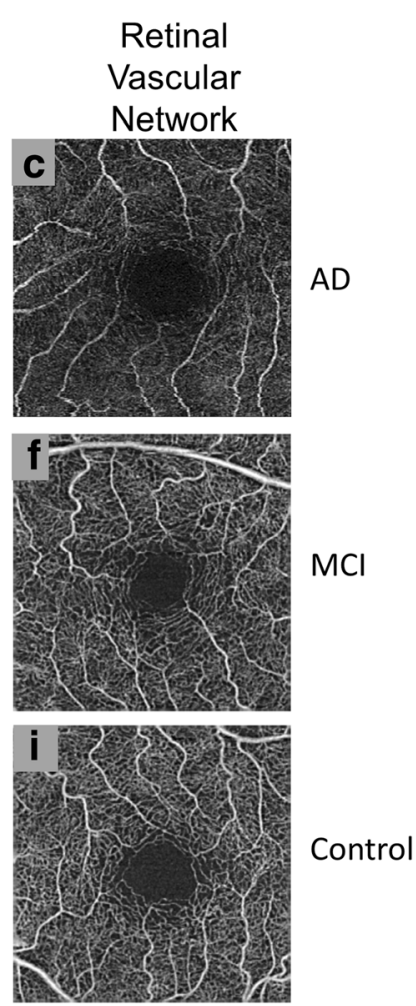

Retinal

cular

$\mathrm{Cl}$

Control

Fig. 5 Optical coherence tomography angiography (OCTA) of the parafoveal region in Mild Cognitive Impairment (MCI) and Alzheimer's Disease (AD). As determined using Cirrus ${ }^{\mathrm{T}}$, retinal microvasculature for patients with $\mathrm{AD}(\mathbf{a}, \mathbf{b}$, and $\mathbf{c}), \mathrm{MCl}(\mathbf{d}, \mathbf{e}$, and $\mathbf{f})$, and healthy controls $(\mathbf{g}, \mathbf{h}$, and $\mathbf{i})$ are shown. For the large vessels, no significant differences in density were observed, but some degree of increased tortuosity was seen in the superficial vascular plexus (SVP) (a) in comparison to normal controls (g). The microvasculature of the deep vascular plexus (DVP) (b) had a significant decrease in density with a visually larger foveal avascular zone when compared with normal controls (h) and $\mathrm{MCl}$ patients (e). The overall retinal vascular network contains both the SVP and DVP (c, f, and i). Bar = $0.5 \mathrm{~mm}$ [31]. (Images provided courtesy of Dr. Hong Jiang, MD, PhD of Bascom Palmer Eye Institute at the University of Miami) 
with GCIP and RNFL thicknesses in patients with glaucoma, OCTA might also be an ideal candidate for monitoring NAION progression and identify potential OCTA-based biomarkers for NAION [35, 36].

\section{Other optic neuropathies}

Primarily affecting males between the ages of 15 and 35, Leber's hereditary optic neuropathy (LHON) is a rare genetic disorder inherited through mitochondrial DNA [72]. LHON typically presents as monocular optic atrophy [34] that develops into bilateral central visual field defects within weeks to months after initial symptom onset. The characteristic microangiography of LHON accompanied by RNFL thinning has been non-invasively demonstrated by OCTA as increased tortuosity and decrease in observable vessels in the peripapillary microvasculature. The reduced visibility of microvessels indicates thinning of the vasculature with decreased vessel density, which could theoretically relate to lowered metabolic demand in the setting of RNFL/axonal degeneration. Alternatively, it is possible that the pathobiology of LHON may directly include destruction of the microvessels, although this remains to be elucidated [34]. Further larger studies using OCTA may help to delineate the role of microvascular aberrations as part of the LHON disease process.
Resulting from elevated intra-cranial pressure that may be idiopathic in etiology or secondary to numerous causes including but not limited to intracranial masses for example, papilledema -is swelling of the optic discs - that usually occurs bilaterally. Vision loss is uncommon in early papilledema, but decreased visual acuity and visual field loss is often profound in advanced papilledema [73]. Using OCT, papilledema has also been shown to be associated with a significant increase in disc volume due to overall swelling, but also degeneration of the inner retinal layers in the setting of chronic papilledema [74]. OCTA demonstrated an increased visibility of the peripapillary vascular network in chronic papilledema, which reveal an increased diameter of vessels along with an increase in vessel density [34]. Additional studies with a quantitative measurement of the microvasculature for perfusion density will be necessary to further characterize the role of vascular alternations in the progression of papilledema.

\section{Conclusions}

OCTA is an advanced ophthalmic imaging technique that can non-invasively generate angiograms with depth information for the posterior segment of the eye. The subsequent measures of retinal vascular structure and indirect blood flow enable the detection of alterations
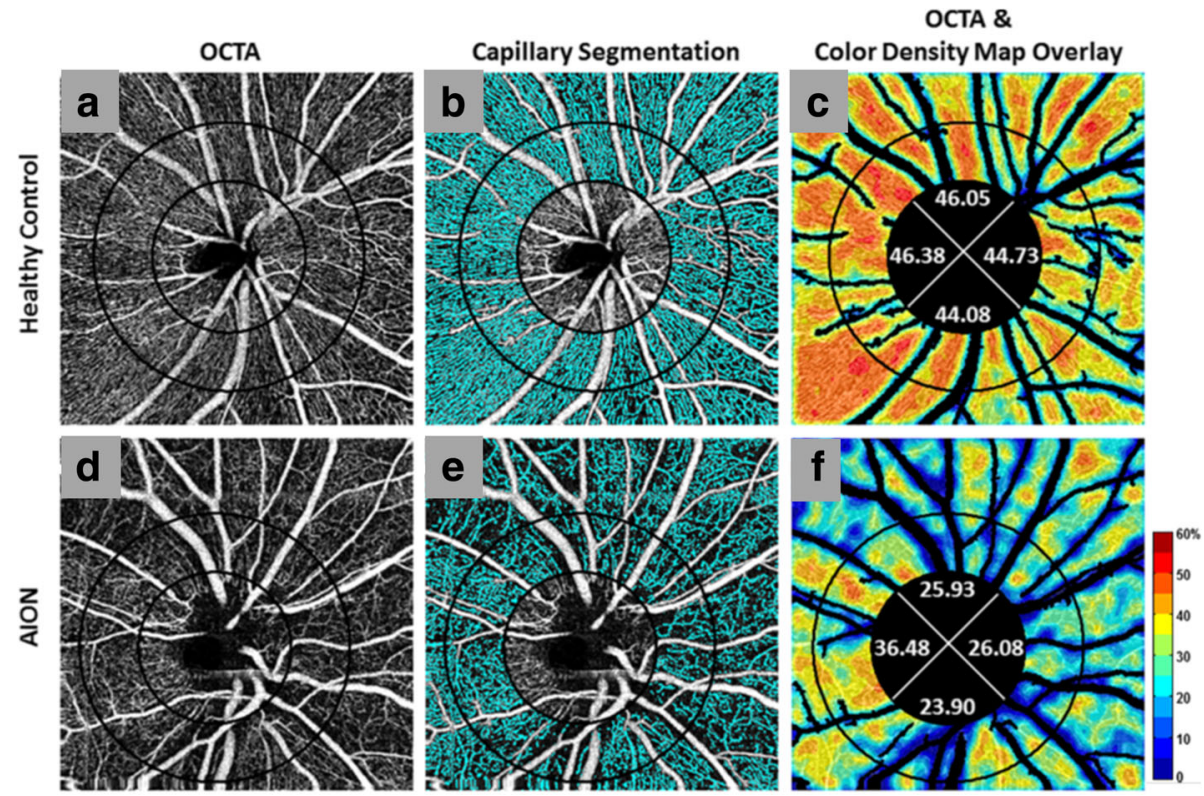

Fig. 6 Optical coherence tomography angiography (OCTA) with peripapillary capillary density maps in Non-arteritic Anterior Ischemic Optic Neuropathy (NAION). Images were derived using Angiovue ${ }^{\text {TM }}$ with SSADA and they depict optic disc-centered angiograms (first column) (a, $\mathbf{d}$ ) with perfused microvessels marked in cyan (second column). The major vessels were not included in the capillary segmentation in panels $\mathbf{b}$ and e. Original OCTA overlaid with corresponding color-coded density map (legend: higher density \% up to $60 \%=$ redder) and capillary density percentage for four quadrants (third column) (c, f). Patients with NAION had a smaller perfusion region when compared with normal controls as seen with the visible loss of microvessels (cyan) in NAION (e) in comparison to healthy controls (b) and the decreased capillary density percentages (less red overall) for all quadrants in NAION (f) in comparison to healthy controls (c). (Reprinted from Fard et al. Pattern of peripapillary capillary density loss in ischemic optic neuropathy compared to that in primary open-angle glaucoma. PLoS One, 13:e0189237 (2018), DOl: https://doi.org/10.1371/journal.pone.0189237) [33] 
in the structure and function of the vasculature in the retina, including the microvasculature that can be qualitatively and quantitatively analyzed. It is worthwhile to note limitations of the OCTA, including the lack of an industry standard for image processing, segmentation of vessel layers, and quantitative analysis. Furthermore, anatomical variables such as individual differences in blood flow and position of vessels may cause variability between subjects, both especially for qualitative and quantitative studies. Nevertheless, while the field of view is relatively small in comparison to other ophthalmic techniques such as FA, ICGA, and RFI [11, 17, 75], significant findings have been identified in a number of potentially sight-threatening ophthalmic disorders [76]. Capable of non-invasively imaging microvascular features that may be obscured or undetectable in other ophthalmic imaging techniques, OCTA utilization is growing across a host of neurological disorders (including cerebrovascular diseases) and contributing towards advancing our understanding of operative disease mechanisms as well as the potential identification of novel biomarkers, which could collectively lead to novel therapeutic targets/ strategies in the future. Blood flow or vessel density was reported to be decreased in MS, AD, and $\mathrm{ON}$ in various regions of the posterior segment vasculature. These emerging research findings suggest a role for vascular alterations in the pathobiology of these diseases, although it is unclear if and to what magnitude these changes may contribute to disease progression. Further investigations with larger sample sizes, assessment of greater regions of the vasculature, and additional OCTA parameters may help to characterize these neurological disorders and determine the true utility of OCTA in the field of neurology.

\section{Acknowledgements}

We thank Dr. Hong Jiang MD, PhD, of the Bascom Palmer Eye Institute at the University of Miami for providing the images used for Fig. 5.

\section{Funding}

This review article was supported in part by a grant (RG-1606-08768) from the National Multiple Sclerosis Society to SS.

\section{Disclosures}

Peter Calabresi has received personal honorariums for consulting from Biogen and Disarm Therapeutics. He is PI on research grants to Johns Hopkins from Medlmmune, Annexon, and Genzyme.

Shiv Saidha has received consulting fees from Medical Logix for the development of CME programs in neurology and has served on scientific advisory boards for Biogen-ldec, Genzyme, Genentech Corporation, EMD Serono \& Novartis. He is the $\mathrm{Pl}$ of investigator-initiated studies funded by Genentech Corporation and Biogen Idec, and received support from the Race to Erase MS foundation. He has received equity compensation for consulting from JuneBrain LLC, a retinal imaging device developer. He is also the site investigator of a trial sponsored by MedDay Pharmaceuticals.

Liang Wang, Olwen Murphy, and Natalia Gonzalez Caldito do not have any disclosures.

\section{Authors' contributions}

LW and SS were the major contributors in writing the manuscript. All authors read and approved the final manuscript.

Ethics approval and consent to participate

Not applicable.

\section{Competing interests}

The authors declare that they have no competing interests.

\section{Author details}

'Department of Biology, Johns Hopkins University, Krieger School of Arts and Sciences, Baltimore, MD, USA. ${ }^{2}$ Division of Neuroimmunology and Neurological Infections, Johns Hopkins Hospital, 600 N. Wolfe St., Baltimore, MD 21287, USA

Received: 9 February 2018 Accepted: 27 April 2018

Published online: 12 May 2018

\section{References}

1. Carpineto P, Mastropasqua R, Marchini G, Toto L, Di Nicola M, Di Antonio L. Reproducibility and repeatability of foveal avascular zone measurements in healthy subjects by optical coherence tomography angiography. Br J Ophthalmol. 2016;100(5):671-6.

2. Huang $Y$, Zhang $Q$, Thorell MR, An L, Durbin MK, Laron M, et al. Swept-source OCT angiography of the retinal vasculature using intensity differentiationbased optical microangiography algorithms. Ophthalmic Surg Lasers Imaging Retina. 2014;45(5):382-9.

3. Nehemy MB, Brocchi DN, Veloso CE. Optical Coherence Tomography Angiography Imaging of Quiescent Choroidal Neovascularization in AgeRelated Macular Degeneration. Ophthalmic Surg Lasers Imaging Retina. 2015:46(10):1056-7.

4. Rispoli M, Savastano MC, Lumbroso B. Capillary network anomalies in branch retinal vein occlusion on optical coherence tomogrpahy angiography. Retina. 2015;35(11):2332-8.

5. de Carlo TE, Chin AT, Joseph T, Baumal CR, Witkin AJ, Duker JS, et al. Distinguishing Diabetic Macular Edema From Capillary Nonperfusion Using Optical Coherence Tomography Angiography. Ophthalmic Surg Lasers Imaging Retina. 2016;47(2):108-14.

6. Dansingani KK, Freund KB. Optical Coherence Tomography Angiography Reveals Mature, Tangled Vascular Networks in Eyes With Neovascular AgeRelated Macular Degeneration Showing Resistance to Geographic Atrophy. Ophthalmic Surg Lasers Imaging Retina. 2015:46(9):907-12.

7. Jia Y, Wei E, Wang X, Zhang X, Morrison JC, Parikh M, et al. Optical coherence tomography angiography of optic disc perfusion in glaucoma. Ophthalmology. 2014;121(7):1322-32.

8. Jia Y, Bailey ST, Wilson DJ, Tan O, Klein ML, Flaxel CJ, et al. Quantitative optical coherence tomography angiography of choroidal neovascularization in age-related macular degeneration. Ophthalmology. 2014;121(7):1435-44.

9. Balaratnasingam C, Yannuzzi LA, Spaide RF. Possbile choroidal neovacularization in macular telangiectasia type 2. Retina. 2015;35(11):2317-22.

10. Coscas F, Glacet-Bernard A, Miere A, Caillaux V, Uzzan J, Lupidi M, et al. Optical Coherence Tomography Angiography in Retinal Vein Occlusion: Evaluation of Superficial and Deep Capillary Plexa. Am J Ophthalmol. 2016; 161:160-71

11. de Castro-Abeger AH, de Carlo TE, Duker JS, Baumal CR. Optical Coherence Tomography Angiography Compared to Fluorescein Angiography in Branch Retinal Artery Occlusion. Ophthalmic Surg Lasers Imaging Retina. 2015; 46(10):1052-4.

12. Couturier A, Mané V, Bonnin S, Erginay A, Massin P, Gaudric A, et al. Capillary plexus anomalies in diabetic retinopathy on optical coherence tomography angiography. Retina. 2015;35(11):2384-91.

13. Hwang TS, Jia Y, Gao SS, Bailey ST, Lauer AK, Flaxel CJ, et al. Optical coherence tomography angiography features of diabetic retinopathy. Retina. 2015;35(11):2371-6.

14. Matsunaga D, Yi J, Puliafito CA, Kashani AH. OCT angiography in healthy human subjects. Ophthalmic Surg Lasers Imaging Retina. 2014:45(6):510-5.

15. Stanga PE, Lim Jl, Hamilton P. Indocyanine green angiography in chorioretinal diseases: indications and interpretation: an evidence-based update. Ophthalmology. 2003;110(1):15-21. 
16. Ito YN, Mori K, Young-Duvall J, Yoneya S. Aging changes of the choroidal dye filling pattern in indocyanine green angiography of normal subjects. Retina. 2001;21(3):237-42

17. Teussink MM, Breukink MB, van Grinsven MJ, Hoyng CB, Klevering BJ, Boon CJ, et al. OCT Angiography Compared to Fluorescein and Indocyanine Green Angiography in Chronic Central Serous Chorioretinopathy. Invest Ophthalmol Vis Sci. 2015;56(9):5229-37.

18. Wei Y, Jiang H, Shi Y, Qu D, Gregori G, Zheng F, et al. Age-Related Alterations in the Retinal Microvasculature, Microcirculation, and Microstructure. Invest Ophthalmol Vis Sci. 2017:58(9):3804-17.

19. Wang RK, Hurst S. Mapping of cerebro-vascular blood perfusion in mice with skin and skull intact by Optical Micro-AngioGraphy at 1.3 mum wavelength. Opt Express. 2007;15(18):11402-12.

20. Nakazawa T. Ocular Blood Flow and Influencing Factors for Glaucoma. Asia Pac J Ophthalmol (Phila). 2016;5(1):38-44.

21. Levison AL, Baynes K, Lowder CY, Srivastava SK. OCT Angiography Identification of Choroidal Neovascularization Secondary to Acute Zonal Occult Outer Retinopathy. Ophthalmic Surg Lasers Imaging Retina. 2016;47(1):73-5.

22. London A, Benhar I, Schwartz M. The retina as a window to the brain-from eye research to CNS disorders. Nat Rev Neurol. 2013;9(1):44-53.

23. Patton N, Aslam T, Macgillivray T, Pattie A, Deary IJ, Dhillon B. Retinal vascular image analysis as a potential screening tool for cerebrovascular disease: a rationale based on homology between cerebral and retinal microvasculatures. J Anat. 2005;206(4):319-48.

24. Kur J, Newman EA, Chan-Ling T. Cellular and physiological mechanisms underlying blood flow regulation in the retina and choroid in health and disease. Prog Retin Eye Res. 2012;31(5):377-406.

25. Wang X, Jia Y, Spain R, Potsaid B, Liu JJ, Baumann B, et al. Optical coherence tomography angiography of optic nerve head and parafovea in multiple sclerosis. Br J Ophthalmol. 2014;98(10):1368-73.

26. Feucht N, Maier M, Lepennetier G, Pettenkofer M, Wetzlmair C, Daltrozzo T, et al. Optical coherence tomography angiography indicates associations of the retinal vascular network and disease activity in multiple sclerosis. Mult Scler. 2018; 1352458517750009. https://doi.org/10.1177/1352458517750009.

27. Lanzillo R, Cennamo G, Criscuolo C, Carotenuto A, Velotti N, Sparnelli F, et al. Optical coherence tomography angiography retinal vascular network assessment in multiple sclerosis. Mult Scler. 2017; 1352458517729463. https://doi.org/10.1177/1352458517729463.

28. Spain RI, Liu L, Zhang X, Jia Y, Tan O, Bourdette D, et al. Optical coherence tomography angiography enhances the detection of optic nerve damage in multiple sclerosis. Br J Ophthalmol. 2018;102:520-4.

29. Bulut M, Kurtuluş F, Gözkaya O, Erol MK, Cengiz A, Akidan M, et al. Evaluation of optical coherence tomography angiographic findings in Alzheimer's type dementia. Br J Ophthalmol. 2018;102(2):233-7.

30. den Haan J, Janssen SF, van de Kreeke JA, Scheltens P, Verbraak FD, Bouwman FH. Retinal thickness correlates with parietal cortical atrophy in early-onset Alzheimer's disease and controls. Alzheimers Dement (Amst). 2017:10:49-55

31. Jiang $H$, Wei $Y$, Shi $Y$, Wright $C B$, Sun $X$, Gregori $G$, et al. Altered Macular Microvasculature in Mild Cognitive Impairment and Alzheimer Disease. J Neuroophthalmol. 2017; https://doi.org/10.1097/WNO.0000000000000580.

32. Balducci N, Morara M, Veronese C, Barboni P, Casadei NL, Savini G, et al. Optical coherence tomography angiography in acute arteritic and nonarteritic anterior ischemic optic neuropathy. Graefes Arch Clin Exp Ophthalmol. 2017;255(11):2255-61.

33. Fard MA, Suwan Y, Moghimi S, Geyman LS, Chui TY, Rosen RB, et al. Pattern of peripapillary capillary density loss in ischemic optic neuropathy compared to that in primary open-angle glaucoma. PLoS One. 2018;13(1):e0189237.

34. Ghasemi Falavarjani K, Tian JJ, Akil H, Garcia GA, Sadda SR, Sadun AA. Swept-source optical coherence tomography angiography of the optic disk in optic neuropathy. Retina. 2016;36(Suppl 1):S168-77.

35. Ling JW, Yin X, Lu QY, Chen YY, Lu PR. Optical coherence tomography angiography of optic disc perfusion in non-arteritic anterior ischemic optic neuropathy. Int J Ophthalmol. 2017;10(9):1402-6.

36. Song Y, Min JY, Mao L, Gong YY. Microvasculature dropout detected by the optical coherence tomography angiography in nonarteritic anterior ischemic optic neuropathy. Lasers Surg Med. 2018;50:194-201

37. Zhang Q, Lee CS, Chao J, Chen CL, Zhang T, Sharma U, et al. Wide-field optical coherence tomography based microangiography for retinal imaging. Sci Rep. 2016;6:22017.
38. Jia Y, Bailey ST, Hwang TS, McClintic SM, Gao SS, Pennesi ME, et al. Quantitative optical coherence tomography angiography of vascular abnormalities in the living human eye. Proc Natl Acad Sci U S A. 2015;112(18):E2395-402.

39. Moussa M, Leila M, Khalid H, Lolah M. Detection of Silent Type I Choroidal Neovascular Membrane in Chronic Central Serous Chorioretinopathy Using En Face Swept-Source Optical Coherence Tomography Angiography. J Ophthalmol. 2017;2017:6913980. https://doi.org/10.1155/2017/6913980.

40. Munk MR, Giannakaki-Zimmermann H, Berger L, Huf W, Ebneter A, Wolf S, et al. OCT-angiography: A qualitative and quantitative comparison of 4 OCT-A devices. PLoS One. 2017;12(5):e0177059.

41. Zhang Q, Wang RK, Chen CL, Legarreta AD, Durbin MK, An L, et al. Swept source optical coherence tomography angiography of neovascular macular telangiectasia type 2. Retina. 2015;35(11):2285-99.

42. Miller AR, Roisman L, Zhang Q, Zheng F, Rafael de Oliveira Dias J, Yehoshua $Z$, et al. Comparison Between Spectral-Domain and Swept-Source Optical Coherence Tomography Angiographic Imaging of Choroidal Neovascularization. Invest Ophthalmol Vis Sci. 2017;58(3):1499-505.

43. Zhang Q, Huang Y, Zhang T, Kubach S, An L, Laron M, et al. Wide-field imaging of retinal vasculature using optical coherence tomographybased microangiography provided by motion tracking. J Biomed Opt. 2015:20(6):066008.

44. Chen $\mathrm{CL}$, Wang RK. Optical coherence tomography based angiography [Invited]. Biomed Opt Express. 2017;8(2):1056-82.

45. de Carlo TE, Bonini Filho MA, Baumal CR, Reichel E, Rogers A, Witkin AJ, et al. Evaluation of Preretinal neovascularization in proliferative diabetic retinopathy using optical coherence tomography angiography. Ophthalmic Surg Lasers Imaging Retina. 2016;47(2):115-9.

46. Chen Z, Huang D, Izatt JA, Wang RK, Werner JS, Yasuno Y. Re: Spaide et al.: Volume-rendering optical coherence tomography angiography of macular telangiectasia type 2 (Ophthalmology 2015;122:2261-9). Ophthalmology. 2016;123(3):e24

47. Wang $X$, Jiang C, Ko T, Kong X, Yu X, Min W, et al. Correlation between optic disc perfusion and glaucomatous severity in patients with open-angle glaucoma: an optical coherence tomography angiography study. Graefes Arch Clin Exp Ophthalmol. 2015;253(9):1557-64.

48. Choi W, Mohler KJ, Potsaid B, Lu CD, Liu JJ, Jayaraman V, et al. Choriocapillaris and choroidal microvasculature imaging with ultrahigh speed OCT angiography. PLoS One. 2013;8(12):e81499.

49. Yu J, Jiang C, Wang X, Zhu L, Gu R, Xu H, et al. Macular perfusion in healthy Chinese: an optical coherence tomography angiogram study. Invest Ophthalmol Vis Sci. 2015;56(5):3212-7.

50. Grunwald JE, Piltz J, Patel N, Bose S, Riva CE. Effect of aging on retinal macular microcirculation: a blue field simulation study. Invest Ophthalmol Vis Sci. 1993;34(13):3609-13.

51. Emeterio Nateras OS, Harrison JM, Muir ER, Zhang Y, Peng Q, Chalfin S, et al. Choroidal blood flow decreases with age: an MRI study. Curr Eye Res. 2014; 39(10):1059-67.

52. Mansour AM, Schachat A, Bodiford G, Haymond R. Foveal avascular zone in diabetes mellitus. Retina. 1993;13(2):125-8.

53. Moghimi S, Hosseini H, Riddle J, Lee GY, Bitrian E, Giaconi J, et al. Measurement of optic disc size and rim area with spectral-domain OCT and scanning laser ophthalmoscopy. Invest Ophthalmol Vis Sci. 2012;53(8):4519-30.

54. Gadde SG, Anegondi N, Bhanushali D, Chidambara L, Yadav NK, Khurana A, et al. Quantification of Vessel Density in Retinal Optical Coherence Tomography Angiography Images Using Local Fractal Dimension. Invest Ophthalmol Vis Sci. 2016;57(1):246-52.

55. Chan G, Balaratnasingam C, Yu PK, Morgan WH, McAllister IL, Cringle SJ, et al. Quantitative morphometry of perifoveal capillary networks in the human retina. Invest Ophthalmol Vis Sci. 2012;53(9):5502-14.

56. Fujiwara A, Morizane $Y$, Hosokawa M, Kimura S, Shiode $Y$, Hirano M, et al. Factors affecting foveal avascular zone in healthy eyes: An examination using swept-source optical coherence tomography angiography. PLoS One. 2017;12(11):e0188572.

57. Ikuta F, Zimmerman HM. Distribution of plaques in seventy autopsy cases of multiple sclerosis in the United States. Neurology. 1976;26(6 PT 2):26-8.

58. Toussaint D, Périer O, Verstappen A, Bervoets S. Clinicopathological study of the visual pathways, eyes, and cerebral hemispheres in 32 cases of disseminated sclerosis. J Clin Neuroophthalmol. 1983:3(3):211-20.

59. Saidha S, Al-Louzi O, Ratchford JN, Bhargava P, Oh J, Newsome SD, et al. Optical coherence tomography reflects brain atrophy in multiple sclerosis: A four-year study. Ann Neurol. 2015;78(5):801-13. 
60. Plumb J, McQuaid S, Mirakhur M, Kirk J. Abnormal endothelial tight junctions in active lesions and normal-appearing white matter in multiple sclerosis. Brain Pathol. 2002;12(2):154-69.

61. Doche E, Lecocq A, Maarouf A, Duhamel G, Soulier E, Confort-Gouny S, et al. Hypoperfusion of the thalamus is associated with disability in relapsing remitting multiple sclerosis. J Neuroradiol. 2017:44(2):158-64.

62. Narayana PA, Zhou Y, Hasan KM, Datta S, Sun X, Wolinsky JS. Hypoperfusion and T1-hypointense lesions in white matter in multiple sclerosis. Mult Scler. 2014;20(3):365-73.

63. Jiang H, Delgado S, Tan J, Liu C, Rammohan KW, DeBuc DC, et al. Impaired retinal microcirculation in multiple sclerosis. Mult Scler. 2016;22(14):1812-20.

64. Zeis T, Graumann U, Reynolds R, Schaeren-Wiemers N. Normal-appearing white matter in multiple sclerosis is in a subtle balance between inflammation and neuroprotection. Brain. 2008;131(Pt 1):288-303.

65. Alzheimer's Association. 2015 Alzheimer's disease facts and figures. Alzheimers Dement. 2015;11(3):332-84.

66. Budson AE, Solomon PR. New diagnostic criteria for Alzheimer's disease and mild cognitive impairment for the practical neurologist. Pract Neurol. 2012; 12(2):88-96.

67. Vos SJ, Verhey F, Frölich L, Kornhuber J, Wiltfang J, Maier W, et al. Prevalence and prognosis of Alzheimer's disease at the mild cognitive impairment stage. Brain. 2015:138(Pt 5):1327-38.

68. Yamashita Kl, Taniwaki Y, Utsunomiya H, Taniwaki T. Cerebral blood flow reduction associated with orientation for time in amnesic mild cognitive impairment and Alzheimer disease patients. J Neuroimaging. 2014;24(6):590-4.

69. Snyder PJ, Johnson LN, Lim YY, Santos CY, Alber J, Maruff P, et al. Nonvascular retinal imaging markers of preclinical Alzheimer's disease. Alzheimers Dement (Amst). 2016:4:169-78.

70. Garcia-Martin E, Bambo MP, Marques ML, Satue M, Otin S, Larrosa JM, et al. Ganglion cell layer measurements correlate with disease severity in patients with Alzheimer's disease. Acta Ophthalmol. 2016;94(6):e454-9.

71. Akbari M, Abdi P, Fard MA, Afzali M, Ameri A, Yazdani-Abyaneh A, et al. Retinal Ganglion Cell Loss Precedes Retinal Nerve Fiber Thinning in Nonarteritic Anterior Ischemic Optic Neuropathy. J Neuroophthalmol. 2016; 36(2):141-6.

72. Milea D, Verny C. [Hereditary optic neuropathies]. Rev Neurol (Paris). 2012; 168(10):706-9.

73. Friedman DI. Papilledema and idiopathic intracranial hypertension. Continuum (Minneap Minn). 2014;20(4 Neuro-ophthalmology):857-76.

74. Goldhagen BE, Bhatti MT, Srinivasan PP, Chiu SJ, Farsiu S, El-Dairi MA. Retinal atrophy in eyes with resolved papilledema detected by optical coherence tomography. J Neuroophthalmol. 2015;35(2):122-6.

75. Chhablani J, Bartsch DU, Cheng L, Gomez L, Alshareef RA, Rezeq SS, et al. Segmental reproducibility of retinal blood flow velocity measurements using retinal function imager. Graefes Arch Clin Exp Ophthalmol. 2013; 251(12):2665-70.

76. de Carlo TE, Romano A, Waheed NK, Duker JS. A review of optical coherence tomography angiography (OCTA). Int J Retina Vitreous. 2015;1:5.

\section{Ready to submit your research? Choose BMC and benefit from:}

- fast, convenient online submission

- thorough peer review by experienced researchers in your field

- rapid publication on acceptance

- support for research data, including large and complex data types

- gold Open Access which fosters wider collaboration and increased citations

- maximum visibility for your research: over $100 \mathrm{M}$ website views per year

At BMC, research is always in progress.

Learn more biomedcentral.com/submissions 